\title{
Using the Oxford classification of IgA nephropathy to predict long-term outcomes of Henoch-Schönlein purpura nephritis in adults
}

\author{
Chan Ho Kim ${ }^{1}$, Beom Jin Lim $^{2}$, Yoon Sung Bae ${ }^{2}$, Young Eun Kwon ${ }^{1}$, Yung Ly Kim ${ }^{1}$, \\ Ki Heon Nam ${ }^{1}$, Kyoung Sook Park ${ }^{1}$, Seong Yeong An ${ }^{1}$, Hyang Mo Koo ${ }^{1}$, Fa Mee Doh ${ }^{1}$, \\ Mi Jung Lee ${ }^{1}$, Hyung Jung Oh${ }^{1}$, Tae-Hyun Yoo ${ }^{1}$, Shin-Wook Kang ${ }^{1,3}$, Kyu Hun Choi $^{1}$, \\ Hyun Joo Jeong ${ }^{2}$ and Seung Hyeok Han ${ }^{1}$
}

${ }^{1}$ Division of Nephrology, Department of Internal Medicine, College of Medicine, Yonsei University, Seoul, Korea; ${ }^{2}$ Department of Pathology, College of Medicine, Yonsei University, Seoul, Korea and ${ }^{3}$ Severance Biomedical Science Institute, Yonsei University, Brain Korea 21, Seoul, Korea

\begin{abstract}
Recently, there has been emerging concern that crescents, the main histologic feature of Henoch-Schönlein purpura nephritis, merely reflect active inflammation, and may not be useful in predicting long-term outcomes. We therefore conducted a single-center retrospective study to evaluate whether the new Oxford classification of immunoglobulin A nephropathy can be used to predict long-term outcome in patients with Henoch-Schönlein purpura nephritis. We included 61 biopsy-proven patients with Henoch-Schönlein purpura nephritis between January 1991 and August 2010. In addition to the International Study of Kidney Disease in Children classification, pathologic findings were also evaluated by the Oxford classification. Primary outcomes were defined as either the onset of estimated glomerular filtration rate $<60 \mathrm{ml} / \mathrm{min}$ per $1.73 \mathrm{~m}^{2}$ with $\geq 30 \%$ decrease in estimated glomerular filtration rate from baseline or end-stage renal disease. During a median follow-up of 49.3 months, $13(21 \%)$ patients reached the primary end point. A Kaplan-Meier plot showed that renal event-free survival was significantly longer in patients with $<\mathbf{5 0} \%$ crescents than in those with crescents in $\geq \mathbf{5 0} \%$ of glomeruli $(P=0.003)$. Among the components of the Oxford classification, patients with endocapillary hypercellularity $(E 1 ; P=0.016)$ and tubular atrophy/interstitial fibrosis $(T 1 / T 2 ; P=0.018)$ had lower renal survival rates than those with EO and TO. In a multivariate Cox model adjusted for clinical and pathologic factors, $\mathrm{E} 1$ (hazard ratio $=8.91 ; 95 \%$ confidence interval $=1.47-53.88 ; P=0.017$ ) and $\mathrm{T} 1 / \mathrm{T} 2$ (hazard ratio $=8.74$; $95 \%$ confidence interval $=1.40-54.38 ; P=0.020$ ) were independently associated with reaching a primary outcome, whereas the extent of crescentic lesions was not. Our findings suggest that the Oxford classification can be used in predicting long-term outcomes of Henoch-Schönlein purpura nephritis.

Modern Pathology (2014) 27, 972-982; doi:10.1038/modpathol.2013.222; published online 3 January 2014
\end{abstract}

Keywords: crescents; Henoch-Schönlein purpura nephritis; long-term outcome; the Oxford classification

Henoch-Schönlein purpura is an immune-mediated vasculitis associated with immunoglobulin A (IgA) deposition predominantly affecting the skin, joints, gastrointestinal tract, and kidneys. IgA nephropathy, which is characterized by immune deposits predominantly composed of polymeric $\operatorname{IgA}$ in the glomerular mesangium, is the most common

Correspondence: Professor SH Han, MD, PhD, Division of Nephrology, Department of Internal Medicine, College of Medicine, Yonsei University, 50 Yonsei-ro, Seoul, 120-752, Korea. E-mail: hansh@yuhs.ac

Received 6 March 2013; revised 21 October 2013; accepted 22 October 2013; published online 3 January 2014 glomerular disease worldwide. ${ }^{1,2}$ Despite the different clinical features, histologic and immunofluorescence findings in Henoch-Schönlein purpura nephritis are indistinguishable from those of IgA nephropathy. ${ }^{3}$ As such, it has long been speculated that Henoch-Schönlein purpura nephritis and IgA nephropathy share common pathogenetic mechanisms and that they could even be different manifestations of a single disease process. ${ }^{4-6}$

The risk of progression to chronic kidney disease in patients with Henoch-Schönlein purpura nephritis, which ranges from 5 to $20 \%$ in children, ${ }^{7-9}$ seems to be much higher in adults, and is approximately $30 \% .{ }^{9-11}$ The degree of proteinuria, impairment of 
renal function, and hypertension upon initial presentation have been suggested as risk factors for chronic kidney disease in Henoch-Schönlein purpura nephritis, ${ }^{7-12}$ but none have gained widespread acceptance as ideal prognostic factors in clinical practice. It has also been suggested that the risk of developing chronic kidney disease increases with the severity of the histologic lesions at presentation. ${ }^{7,11}$ Histologic lesions of Henoch-Schönlein purpura nephritis have been grouped by the International Study of Kidney Disease in Children classification into six categories (I, II, III, IV, V, and IV) according to the presence and number of crescents. ${ }^{13}$ There is, however, a lack of consensus regarding the utility of crescents as a long-term predictor. Some studies have shown that a high percentage of crescents predicts adverse renal outcomes, ${ }^{7,11}$ while others did not. $.10,14,15$ Therefore, there is need for a reliable, proven tool that can help clinicians more accurately formulate treatment strategies for patients with Henoch-Schönlein purpura nephritis.

Recently, the Oxford classification, a new classification for IgA nephropathy, was proposed by A Working Group of the International IgA Nephropathy Network and the Renal Pathology Society ${ }^{16,17}$ and has been validated in many studies. ${ }^{18-22}$ This group identified four histopathologic features associated with the progression of kidney disease: mesangial hypercellularity score $(\mathrm{M}$; $\mathrm{M} 0 \leq 0.5$, M1 $>0.5$ ), the presence of endocapillary proliferation (E; E0: absent, E1: present) and segmental glomerulosclerosis/adhesion (S; S0: absent, S1: present), and the severity of tubular atrophy/interstitial fibrosis (T; T0 $\leq 25 \%$, T1: $26-50 \%$, T2 >50\%). Given the histopathological similarities between HenochSchönlein purpura nephritis and IgA nephropathy, the Oxford classification may be an alternative for predicting long-term outcomes in HenochSchönlein purpura nephritis. Thus, in this singlecenter retrospective study, we reclassified 61 patients with Henoch-Schönlein purpura nephritis according to the Oxford classification and evaluated its utility in predicting adverse renal outcomes.

\section{Materials and methods}

\section{Patients}

Using electronic medical records at our institution, data for 1649 patients with Henoch-Schönlein purpura between January 1991 and August 2010 were retrieved. After exclusion of patients who did not show proteinuria or microhematuria and those with diabetes or chronic liver disease $(n=1393), 256$ patients were eligible for the study. Among these, we sorted out 92 patients who were aged $\geq 16$ years and pathologically diagnosed Henoch-Schönlein purpura nephritis. A diagnosis of Henoch-Schönlein purpura nephritis was made when hematuria, proteinuria, and/or renal failure was associated with a palpable purpuric eruption or abdominal or joint pains (at least two of these three clinical signs) and predominant mesangial IgA immune deposits confirmed by renal biopsy. ${ }^{10}$ Other exclusion criteria were as follows: follow-up duration $<1$ year $(n=16)$, poor conservation of pathologic material, which could hinder interpretation $(n=8)$, and inadequate number of glomeruli $<8$ to assess severity and distribution of lesions $(n=7) .{ }^{23}$ Therefore, a total of 61 patients were included in the analysis (Figure 1).

\section{Pathologic and Clinical Data Collection}

All renal biopsies were evaluated by one pathologist blinded to the case histories. To analyze the predictive value of crescents, biopsy specimens were graded according to the International Study of Kidney Disease in Children classification: (I) minimal glomerular abnormalities, (II) mesangial proliferation, (III) mesangial proliferation with crescents in $<50 \%$ of glomeruli, (IV) mesangial proliferation with crescents in 50-75\% of glomeruli, (V) mesangial proliferation with crescents in $>75 \%$ of glomeruli, and (VI) membranoproliferative-like lesions. Because there was no patient with class VI, the histologic findings of the International Study of Kidney Disease in Children classification were categorized into two groups according to the percentage of crescents; group I encompassed classes I, II, and III with crescents in less than $50 \%$ of glomeruli and group II included classes IV and V with crescents in $\geq 50 \%$ of glomeruli. Pathologic findings were then reclassified using the Oxford classification scoring system as previously described. ${ }^{16,17}$ Representative images of each lesion of the Oxford classification are illustrated in Figure 2. Demographic and clinical data, including age at the time of biopsy, gender, medical history, systolic and diastolic blood pressures, follow-up duration, and any treatment with immunosuppressive agents were reviewed from medical records. Hypertension was defined as systolic blood pressure $\geq 140 \mathrm{mmHg}$ and/or diastolic blood pressure $\geq 90 \mathrm{~mm} \mathrm{Hg}$ and/or receiving any antihypertensive medications. Laboratory data such as serum creatinine, serum IgA, 24-h proteinuria, and urinary protein-to-creatinine ratio were also collected. The estimated glomerular filtration rate was calculated using the Chronic Kidney Disease Epidemiology Collaboration equation. ${ }^{24}$

\section{Study Outcomes}

Patients were followed up until their last recorded visit. For patients who died, cause of death and renal function at the time of death were determined. When patients were lost to follow-up, their renal function at last visit was recorded. The primary end 


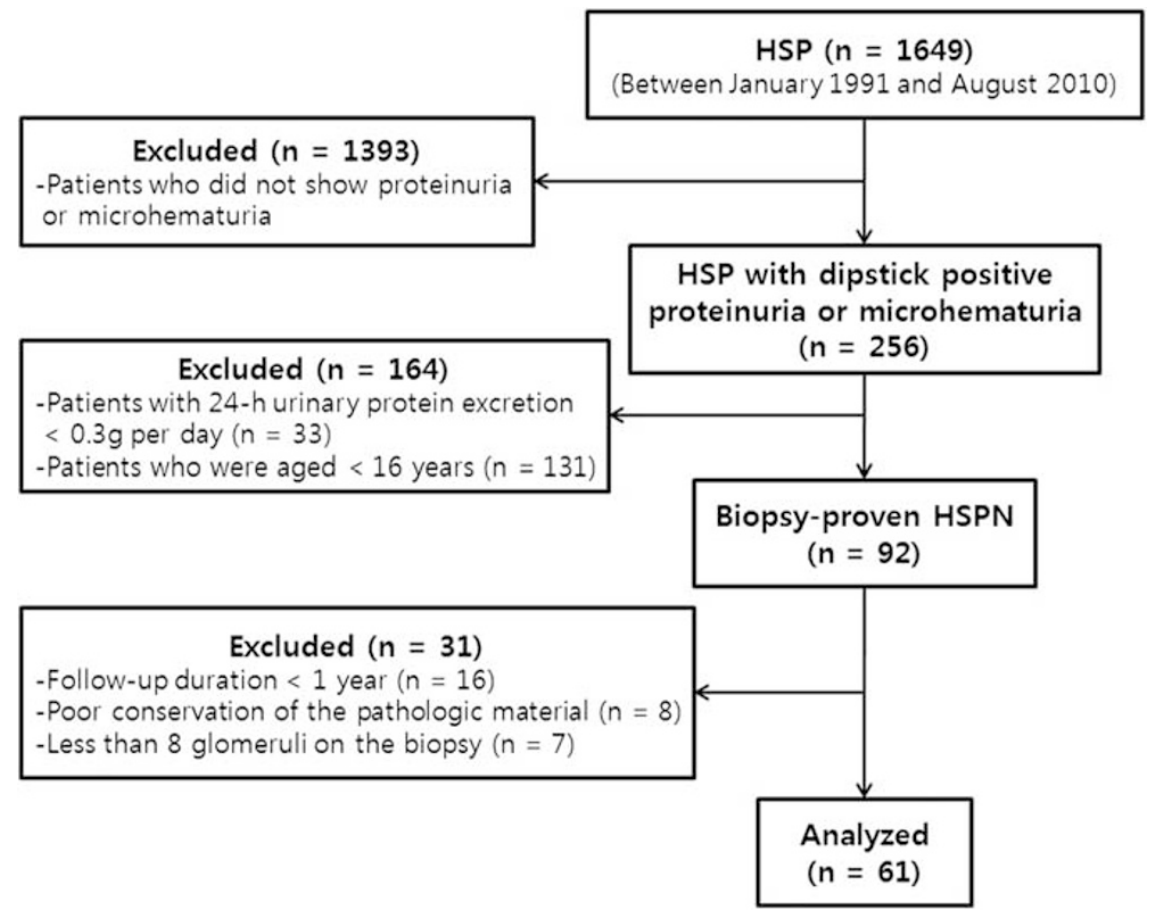

Figure 1 Flow diagram of the study subjects.

point of our study was either the onset of estimated glomerular filtration rate $<60 \mathrm{ml} / \mathrm{min}$ per $1.73 \mathrm{~m}^{2}$ with $\geq 30 \%$ decrease in estimated glomerular filtration rate from baseline or the end-stage renal disease, requiring renal replacement therapy. Patients who reached the primary end point were considered 'progressors'. Renal outcomes were classified as remission, persistent proteinuria, and/or hematuria without renal insufficiency, moderate or severe renal insufficiency, and end-stage renal disease. Remission was defined as normal renal function without proteinuria and microhematuria. In addition, we defined persistent proteinuria as urinary protein-to-creatinine ratio $\geq 0.2 \mathrm{mg} / \mathrm{mg}$, hematuria as $\geq 3$ red blood cells per high-power field, renal insufficiency as estimated glomerular filtration rate $<60 \mathrm{ml} / \mathrm{min}$ per $1.73 \mathrm{~m}^{2}$, moderate renal function impairment as estimated glomerular filtration rate between 30 and $60 \mathrm{ml} / \mathrm{min}$ per $1.73 \mathrm{~m}^{2}$, and severe renal failure as estimated glomerular filtration rate $<30 \mathrm{ml} / \mathrm{min}$ per $1.73 \mathrm{~m}^{2}$.

\section{Statistical Analyses}

Continuous variables were expressed as means \pm s.d. and categorical variables as numbers with percentages. Comparisons were based on the $t$-test for continuous variables and the $\chi^{2}$ test for categorical variables. The Kolmogorov-Smirnov test was used to analyze the normality of the distribution of parameters. Data that did not show normal distributions were expressed as median and interquartile ranges. These variables were compared using the Mann-Whitney test or Kruskal-Wallis test. Eventfree survival for the onset of estimated glomerular filtration rate $<60 \mathrm{ml} / \mathrm{min}$ per $1.73 \mathrm{~m}^{2}$ with $\geq 30 \%$ reduced estimated glomerular filtration rate or end-stage renal disease was derived using the Kaplan-Meier method, and differences between curves were analyzed by log-rank test. Starting point for survival analysis was the time of biopsy. During the follow-up period, two patients died and four were lost to follow-up. These patients did not reach the primary end point until the last follow-up, thus were censored in the survival analysis. For multivariable analyses, we used the Cox regression model. Variables that were included in the model were the amount of proteinuria, the presence of hypertension, estimated glomerular filtration rate at the time of renal biopsy, the level of crescentic lesions, and the individual lesions of the Oxford classification. The results are presented as hazard ratios with $95 \%$ confidence intervals. All tests were two-sided and a $P$-value of $<0.05$ was considered statistically significant. Statistical analyses were performed using SPSS for Windows, version 19.0 (SPSS, Chicago, IL, USA).

\section{Results}

\section{Patients Characteristics}

Median age of the study subjects was 32 (20-42) years and $54 \%$ were male. Hypertension was 
a
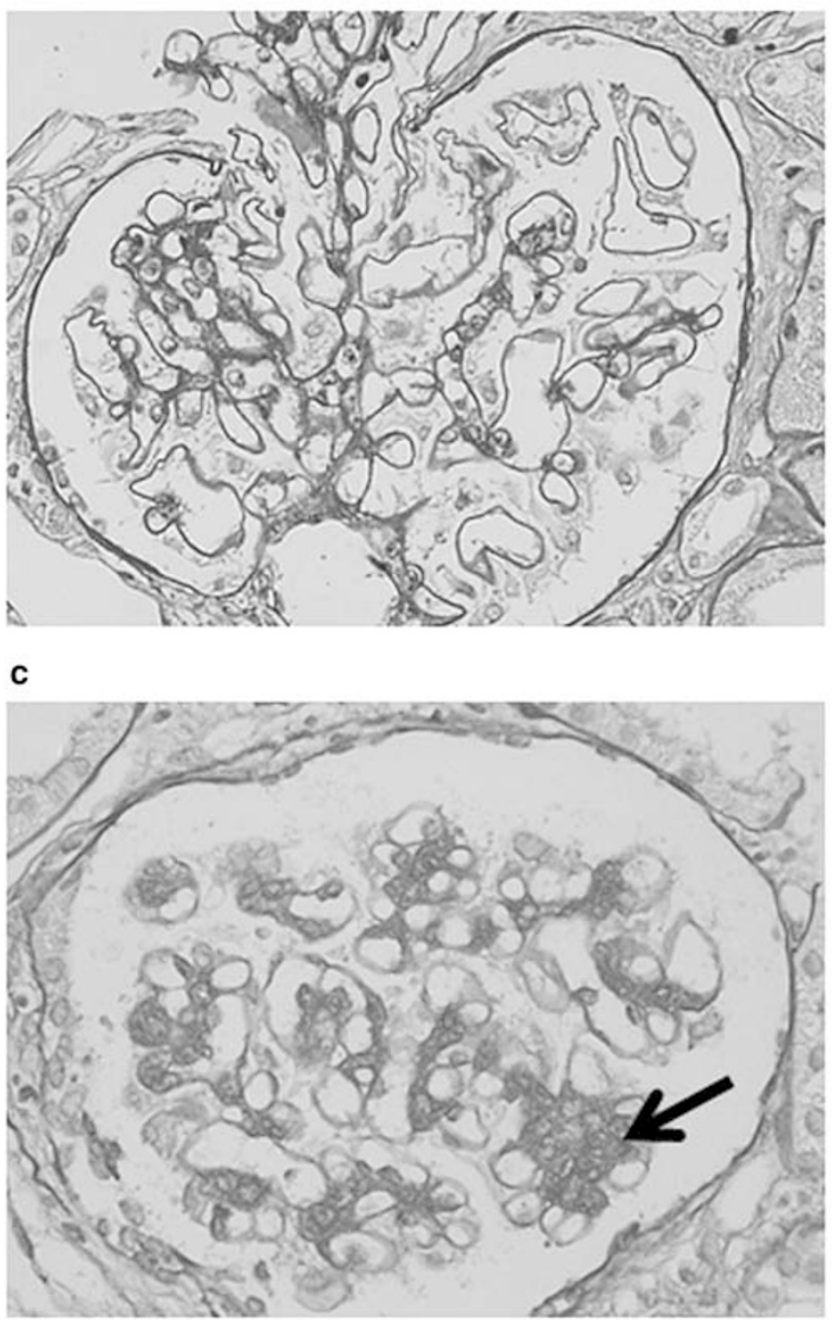

b

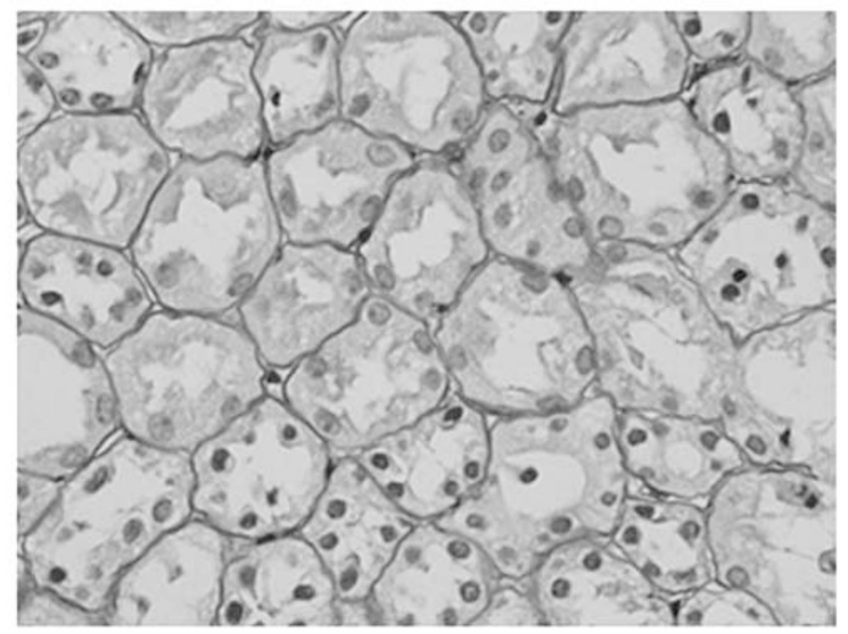

d

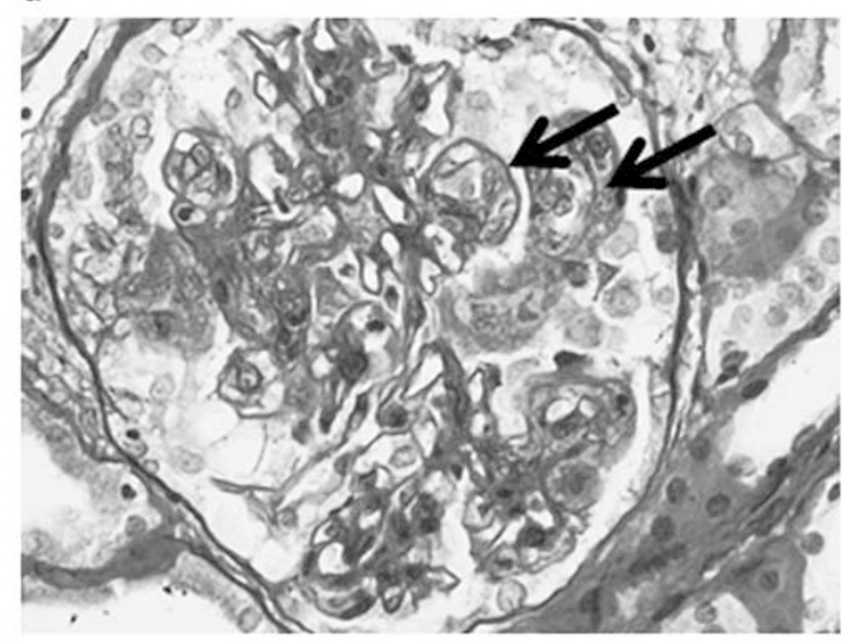

f

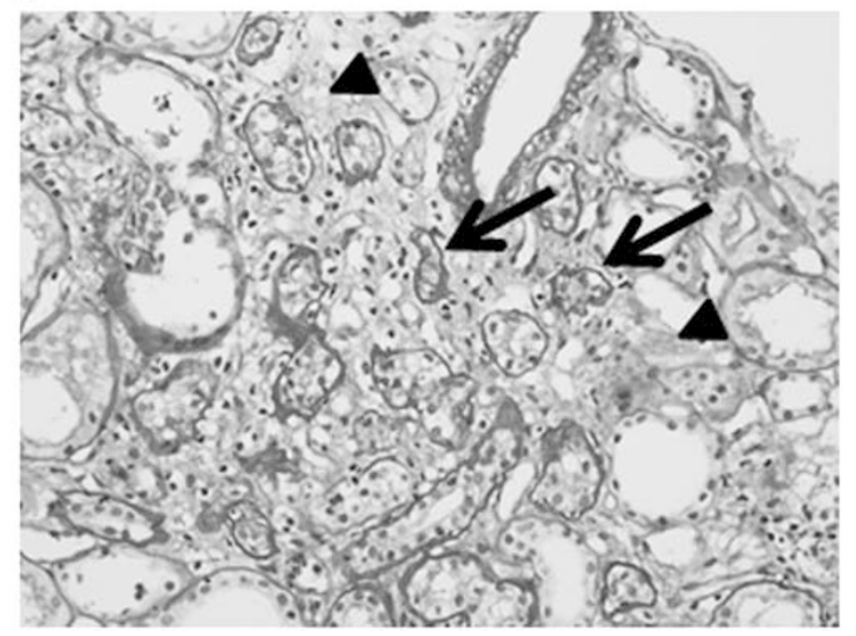

\section{e}

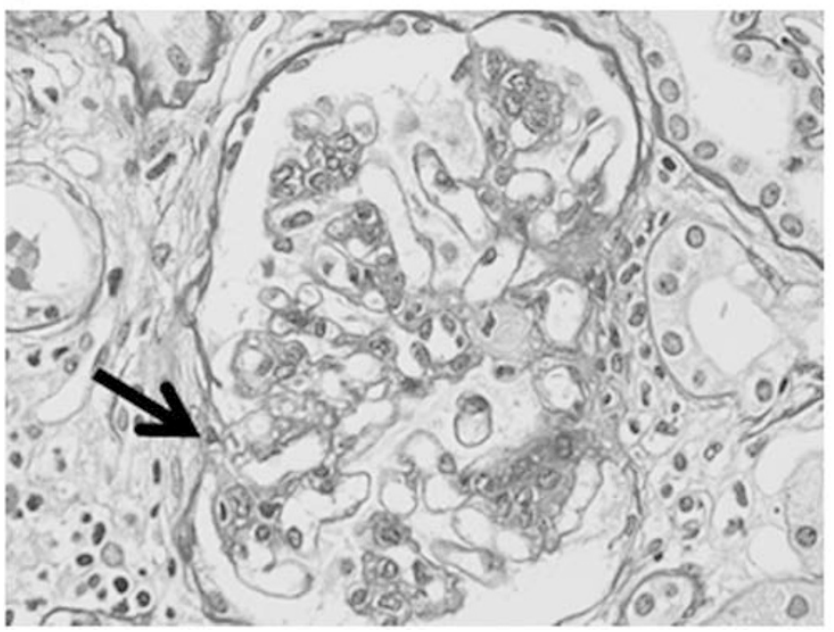

Figure 2 Representative histologic images showing the Oxford classification lesions (mesangial hypercellularity, endocapillary proliferation, segmental glomerulosclerosis/adhesion, and tubular atrophy/interstitial fibrosis). (a) Normal glomerulus $(\times 400)$. (b) Normal tubules $(\times 200)$. (c) Mesangial hypercellularity (arrow, $\times 400)$. More than six mesangial cells per mesangial area are noted, which is compatible with moderate mesangial hypercellularity. (d) Endocapillary proliferation (arrow, $\times 400$ ). Increased number of endothelial cells causes narrowing of the lumen. (e) Segmental glomerulosclerosis/adhesion (arrow, $\times 400$ ). (f) Tubular atrophy (arrow)/interstitial fibrosis (arrowhead, $\times 200)$. When this lesion is $26-50 \%$ and $>50 \%$ of cortical area, it is defined as $\mathrm{T} 1(\mathrm{G}, \times 40)$ and $\mathrm{T} 2(\mathrm{H}, \times 40)$, respectively. 
Table 1 Baseline clinical characteristics according to renal outcome

\begin{tabular}{|c|c|c|c|c|}
\hline Variables & $\begin{array}{c}A l l \\
(\mathrm{n}=61)\end{array}$ & $\begin{array}{l}\text { Non-progressors } \\
(\mathrm{n}=48)\end{array}$ & $\begin{array}{l}\text { Progressors } \\
\quad(\mathrm{n}=13)\end{array}$ & ${ }^{a} \mathrm{P}$-value \\
\hline Age (years) & $34.1 \pm 16.4$ & $33.8 \pm 15.7$ & $35.3 \pm 19.3$ & 0.773 \\
\hline Male gender, $n(\%)$ & $33(54)$ & $25(52)$ & $8(62)$ & 0.544 \\
\hline Hypertension, $n$ (\%) & $18(30)$ & $13(27)$ & $5(39)$ & 0.426 \\
\hline Systolic blood pressure (mm Hg) & $121 \pm 14.8$ & $120 \pm 13.9$ & $125 \pm 17.5$ & 0.249 \\
\hline Diastolic blood pressure (mm Hg) & $76.7 \pm 9.2$ & $76.1 \pm 8.8$ & $78.7 \pm 10.7$ & 0.376 \\
\hline Mean arterial pressure (mm Hg) & $91.5 \pm 9.5$ & $90.8 \pm 8.9$ & $94.3 \pm 11.4$ & 0.242 \\
\hline Body mass index $\left(\mathrm{kg} / \mathrm{m}^{2}\right)$ & $22.3 \pm 3.4$ & $22.0 \pm 3.5$ & $23.3 \pm 3.0$ & 0.259 \\
\hline Gross hematuria, $n(\%)$ & $38(62)$ & $29(60)$ & $9(69)$ & 0.749 \\
\hline \multicolumn{5}{|l|}{ Renal manifestations, n (\%) } \\
\hline Microscopic hematuria with minimal proteinuria $(<0.5 \mathrm{~g} /$ day $)$ & $15(25)$ & $14(30)$ & $1(8)$ & 0.903 \\
\hline Nephritic syndrome with moderate proteinuria $(0.5-3.5 \mathrm{~g} /$ day $)$ & $37(61)$ & $26(54)$ & $11(87)$ & \\
\hline Nephritic-nephrotic syndrome & 9 (15) & $8(17)$ & $1(8)$ & \\
\hline \multicolumn{5}{|l|}{ Extrarenal manifestations, n (\%) } \\
\hline Purpura & $61(100)$ & $48(100)$ & $13(100)$ & - \\
\hline Gastrointestinal symptoms & $25(41)$ & $22(46)$ & $3(23)$ & 0.206 \\
\hline Presence of arthritis/arthralgia & $9(15)$ & $7(15)$ & $2(15)$ & 0.942 \\
\hline \multicolumn{5}{|l|}{ Laboratory measurements } \\
\hline Serum creatinine $(\mathrm{mg} / \mathrm{dl})$ & $0.92 \pm 0.21$ & $0.93 \pm 0.20$ & $0.91 \pm 0.26$ & 0.760 \\
\hline Estimated glomerular filtration rate $\left(\mathrm{ml} / \mathrm{min}\right.$ per $\left.1.73 \mathrm{~m}^{2}\right)$ & $92.6 \pm 22.4$ & $90.7 \pm 20.0$ & $99.9 \pm 29.6$ & 0.304 \\
\hline$\geq 60(n, \%)$ & $56(92)$ & $44(92)$ & $12(92)$ & 0.990 \\
\hline $30-59(n, \%)$ & $5(8)$ & $4(8)$ & $1(8)$ & \\
\hline 24-h urinary protein excretion (g/day) & $1.69 \pm 2.27$ & $1.63 \pm 2.35$ & $1.93 \pm 1.99$ & 0.681 \\
\hline 24-h proteinuria $>3 \mathrm{~g} /$ day $(n, \%)$ & $9(15)$ & $8(17)$ & $1(8)$ & 0.669 \\
\hline Serum IgA level (g/l) & $324 \pm 107$ & $313 \pm 104$ & $362 \pm 116$ & 0.205 \\
\hline Serum albumin level (g/l) & $3.85 \pm 0.75$ & $3.90 \pm 0.74$ & $3.67 \pm 0.78$ & 0.333 \\
\hline Total cholesterol (mg/dl) & $170.4 \pm 47.9$ & $176.7 \pm 48.3$ & $149.7 \pm 41.9$ & 0.074 \\
\hline \multicolumn{5}{|l|}{ Treatments, n (\%) } \\
\hline Renin-angiotensin system blockers & $32(53)$ & $21(44)$ & $11(85)$ & 0.012 \\
\hline Corticosteroids & $30(50)$ & $26(54)$ & $4(31)$ & 0.211 \\
\hline Corticosteroids + other agents ${ }^{b}$ & $7(12)$ & $2(4)$ & $5(39)$ & 0.004 \\
\hline Statin & $10(16)$ & $9(19)$ & $1(8)$ & 0.674 \\
\hline Follow-up duration (months) ${ }^{\mathrm{c}}$ & $49.3(25.2-102.4)$ & $55.0(29.1-104.3)$ & $36.7(16.6-102.0)$ & 0.701 \\
\hline $\begin{array}{l}\text { Duration between the onset of symptoms and } \\
\text { biopsy procedure (days) }{ }^{\mathrm{C}}\end{array}$ & $112(35-293)$ & $92(34-251)$ & $217(113-548)$ & 0.091 \\
\hline
\end{tabular}

The data are expressed as mean \pm s.d. or as numbers (percentages).

${ }^{a} P$-value, comparisons between non-progressors and progressors.

${ }^{\mathrm{b}}$ Other agents include cyclophosphamide, cyclosporine, and azathioprine.

${ }^{\mathrm{C}}$ The data are expressed as median (interquartile range).

present in $18(30 \%)$ patients, median proteinuria was 0.8 (0.25-1.75) g/day, and mean estimated glomerular filtration rate was $92.6 \pm 22.4 \mathrm{ml} / \mathrm{min}$ per $1.73 \mathrm{~m}^{2}$ at the time of biopsy. Median followup duration was $49.3(25.2-102.4)$ months and median interval between clinical presentation and the time of renal biopsy was 112 (35-293) days. Corticosteroids were prescribed to 37 (61\%) patients, and 7 of these patients were also treated with additional immunosuppressants, such as cyclophosphamide, cyclosporine, or azathioprine. The baseline clinical characteristics according to renal outcome are presented in Table 1. No significant differences in clinical characteristics were observed between progressors and non-progressors, with the exception of more frequent use of renin-angiotensin system inhibitors and additional immunosuppressive agents in the former.
Table 2 Frequency of pathologic features of the International Study of Kidney Disease in Children and the Oxford classification in 61 biopsies

\begin{tabular}{|c|c|c|c|c|}
\hline $\begin{array}{l}\text { Histologic } \\
\text { findings }\end{array}$ & $\begin{array}{c}A l l \\
(\mathrm{n}=61), \%\end{array}$ & $\begin{array}{c}\text { Non-progressors } \\
(\mathrm{n}=48), \%\end{array}$ & $\begin{array}{l}\text { Progressors } \\
(\mathrm{n}=13), \%\end{array}$ & ap-value \\
\hline \multicolumn{5}{|c|}{ International Study of Kidney Disease in Children classification } \\
\hline I & $14(23)$ & $14(29)$ & 0 & 0.003 \\
\hline II & $15(25)$ & $15(31)$ & 0 & \\
\hline III & $12(20)$ & $8(17)$ & $4(31)$ & \\
\hline IV & $15(25)$ & $8(17)$ & $7(54)$ & \\
\hline $\mathrm{V}$ & $5(8)$ & $3(6)$ & $2(15)$ & \\
\hline \multicolumn{5}{|c|}{ Oxford classification } \\
\hline M1 & $9(15)$ & $6(13)$ & $3(23)$ & 0.386 \\
\hline E1 & $9(15)$ & $5(10)$ & $4(31)$ & 0.087 \\
\hline $\mathrm{S} 1$ & $21(34)$ & $15(31)$ & $6(46)$ & 0.316 \\
\hline T1 or 2 & $8(13)$ & $5(10)$ & $3(23)$ & 0.350 \\
\hline
\end{tabular}

The data are expressed as numbers (percentages).

${ }^{\mathrm{a}} \mathrm{P}$-value, comparisons between non-progressors and progressors. 
Classification of Histologic Findings According to the International Study of Kidney Disease in Children and the Oxford Classification

In addition to the International Study of Kidney Disease in Children classification, we reclassified the pathologic lesions of patients with Henoch-Schönlein purpura nephritis according to the Oxford classification for IgA nephropathy. The distribution of the International Study of Kidney Disease in Children classification grades and the Oxford classification lesions is shown in Table 2. Crescent formation (grade III, IV, and V) was found in $32(53 \%)$ patients, among whom 20 (33\%) had crescents involved in $\geq 50 \%$ of glomeruli (grade IV and V). The remaining 29 (48\%) patients with grade I and II by the International Study of Kidney Disease in Children classification did not reach the primary end point, and thus they belonged to non-progressors. M1, S1, and E1 were observed in 9 (15\%), 21 (34\%), and 9 $(15 \%)$ patients, respectively. Regarding tubular atrophy/interstitial fibrosis, seven patients $(12 \%)$ had T1 and one $(2 \%)$ had T2. The frequency of pathologic features of Oxford classification was comparable between progressors and non-progressors (Table 2).

Table 3 presents differences in clinical and laboratory findings based on the different pathologic criteria. Patients with E1 had more proteinuria than those with E0, whereas patients with T1/T2 had lower baseline estimated glomerular filtration rate than those with T0. However, blood pressure, estimated glomerular filtration rate, and proteinuria did not differ according to the extent of crescentic lesions, mesangial hypercellularity, or segmental sclerosis.

\section{Renal outcomes}

The renal outcomes in the 61 patients are summarized in Table 4. During follow-up, $46 \%$ of patients had persistent urinary abnormalities with normal renal function and 33\% were in clinical remission, whereas $13(21 \%)$ patients showed an estimated glomerular filtration rate $<60 \mathrm{ml} / \mathrm{min}$ per $1.73 \mathrm{~m}^{2}$ with $\geq 30 \%$ decrease in estimated glomerular filtration rate from baseline. Among these, three developed end-stage renal disease, and of these three, two underwent renal transplantation and one has been on continuous ambulatory peritoneal dialysis for 6 years. No patient progressed to end-stage renal disease before having a $\geq 30 \%$ decrease in estimated glomerular filtration rate.

\section{Prediction of renal outcome}

A Kaplan-Meier plot showed that renal event-free survival was significantly longer in patients with $<50 \%$ crescents than in those with $\geq 50 \%$ crescents $(P=0.003$, Figure 3a). Among components of the

Table 4 Renal outcomes in 61 patients

Renal outcomes, n (\%)

Remission $^{\mathrm{a}}$ $20(33)$

Spontaneous remission ${ }^{\mathrm{b}}$ 7 (12)

Persistent proteinuria and/or hematuria

without renal insufficiency ${ }^{\mathrm{C}}$

Moderate or severe renal insufficiency ${ }^{\mathrm{c}} \quad 13(21)$

End-stage renal disease

The data are expressed as numbers (percentages).

${ }^{a}$ Achieved with immunosuppressive treatment.

${ }^{\mathrm{b}}$ Achieved without any immunosuppressive treatment.

${ }^{\mathrm{C}}$ Renal insufficiency: estimated glomerular filtration rate $<60 \mathrm{ml} / \mathrm{min}$ per $1.73 \mathrm{~m}^{2}$. Moderate renal insufficiency: estimated glomerular filtration rate between 30 and $60 \mathrm{ml} / \mathrm{min}$ per $1.73 \mathrm{~m}^{2}$. Severe renal insufficiency: estimated glomerular filtration rate $<30 \mathrm{ml} / \mathrm{min}$ per $1.73 \mathrm{~m}^{2}$.

Table 3 Comparison clinical features according to extent of crescentic lesions and Oxford classification score at the time of renal biopsy

\begin{tabular}{|c|c|c|c|c|c|c|}
\hline & Mean arterial pressure $(\mathrm{mm} \mathrm{Hg})$ & P-value & $\begin{array}{l}\text { Estimated glomerular filtration rate } \\
\left(\mathrm{ml} / \mathrm{min} \text { per } 1.73 \mathrm{~m}^{2}\right)\end{array}$ & $\mathrm{P}$-value & Proteinuria (g/day) & P-value \\
\hline \multicolumn{7}{|c|}{ Crescents ( $<50 \%$ of glomeruli or $\geq 50 \%$ of glomeruli) } \\
\hline$<50 \%$ & $91.1 \pm 9.6$ & & $92.8 \pm 20.2$ & & $1.61 \pm 2.46$ & \\
\hline$\geq 50 \%$ & $92.4 \pm 9.5$ & 0.633 & $92.2 \pm 27.4$ & 0.924 & $1.88 \pm 1.82$ & 0.660 \\
\hline \multicolumn{7}{|c|}{ Mesangial hypercellularity } \\
\hline Mo & $91.9 \pm 9.7$ & & $90.4 \pm 20.9$ & & $1.76 \pm 2.42$ & \\
\hline M1 & $89.1 \pm 8.4$ & 0.420 & $105.4 \pm 28.0$ & 0.065 & $1.28 \pm 1.00$ & 0.558 \\
\hline \multicolumn{7}{|c|}{ Segmental glomerulosclerosis } \\
\hline So & $92.1 \pm 10.1$ & & $90.5 \pm 19.7$ & & $1.71 \pm 2.59$ & \\
\hline S1 & $90.5 \pm 8.3$ & 0.541 & $96.7 \pm 26.9$ & 0.305 & $1.66 \pm 1.55$ & 0.931 \\
\hline \multicolumn{7}{|c|}{ Endocapillary hypercellularity } \\
\hline E0 & $91.0 \pm 8.4$ & & $94.3 \pm 21.0$ & & $1.20 \pm 1.62$ & \\
\hline E1 & $94.8 \pm 14.4$ & 0.461 & $83.4 \pm 29.3$ & 0.180 & $4.55 \pm 3.34$ & 0.017 \\
\hline \multicolumn{7}{|c|}{ Tubular atrophy/interstitial fibrosis } \\
\hline T0 & $91.4 \pm 9.6$ & & $95.0 \pm 20.4$ & & $1.56 \pm 2.05$ & \\
\hline $\mathrm{T} 1$ or 2 & $92.5 \pm 9.1$ & 0.759 & $77.2 \pm 30.1$ & 0.035 & $2.55 \pm 3.43$ & 0.257 \\
\hline
\end{tabular}

The data are expressed as means \pm s.d. or as numbers (percentages). 
a

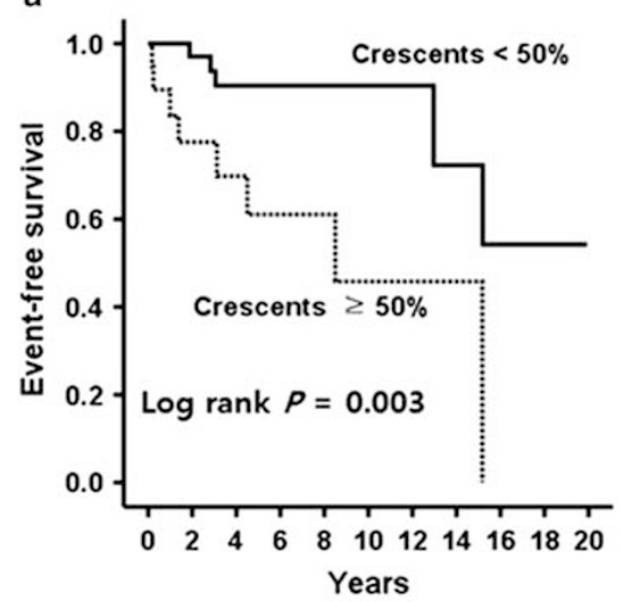

C

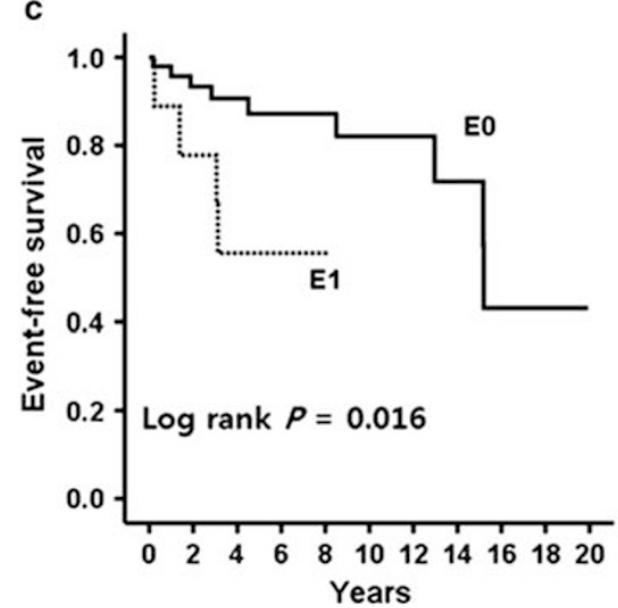

b

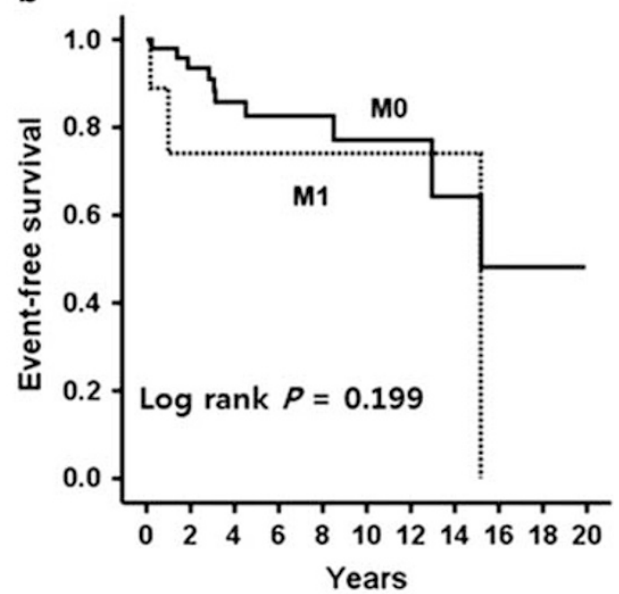

d

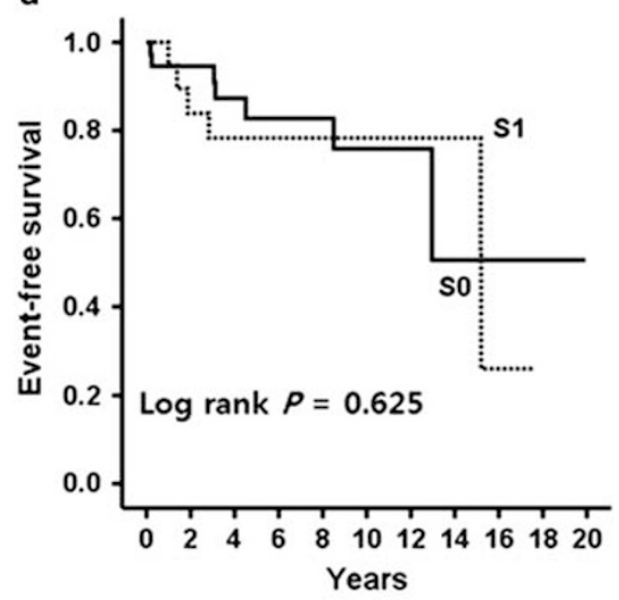

e

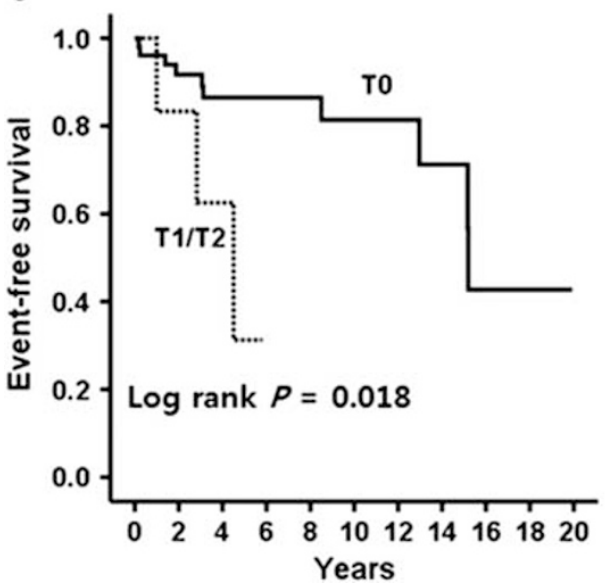

Figure 3 Renal event-free survival according to pathological features. (a) Kaplan-Meier plots for renal event-free survival according to the level of crescentic lesions and (b-e) each lesion of the Oxford classification. Renal event-free survival was significantly longer in patients with $<50 \%$ crescents than in those with $\geq 50 \%$ crescents $(P=0.003)(a)$, among the Oxford classification lesions, E1 $(P=0.016)$ and T1/T2 $(P=0.018)$ were significantly associated with a decreased renal survival $(\mathbf{c}, \mathbf{e})$.

Oxford classification, E1 $(P=0.016)$ and T1/T2 $(P=0.018)$ were significantly associated with a decreased renal survival (Figure 3c and e). Mesangial hypercellularity and segmental sclerosis did not affect renal outcome. Table 5 presents multivariable Cox models adjusted for clinical and pathologic factors. In model 1, crescents were not significantly associated with an increased risk of poor renal 
Table 5 Multivariable Cox models for renal outcomes

\begin{tabular}{|c|c|c|c|c|c|c|}
\hline \multirow{2}{*}{ Variables } & \multicolumn{2}{|l|}{ Model 1} & \multicolumn{2}{|l|}{ Model 2} & \multicolumn{2}{|l|}{ Model 3} \\
\hline & $\begin{array}{l}\text { Hazard ratio (95\% } \\
\text { confidence interval) }\end{array}$ & $\mathrm{P}$-value & $\begin{array}{l}\text { Hazard ratio ( } 95 \% \\
\text { confidence interval) }\end{array}$ & P-value & $\begin{array}{l}\text { Hazard ratio ( } 95 \% \\
\text { confidence interval) }\end{array}$ & $\mathrm{P}$-value \\
\hline Proteinuria (1 g/day) & $1.16(0.86-1.57)$ & 0.134 & $0.89(0.65-1.24)$ & 0.497 & $0.94(0.68-1.31)$ & 0.716 \\
\hline Hypertension (vs no) & $3.54(0.52-23.92)$ & 0.195 & $6.70(0.75-60.23)$ & 0.090 & $2.30(0.18-29.40)$ & 0.522 \\
\hline $\begin{array}{l}\text { Estimated glomerular filtration } \\
\text { rate }\left(1 \mathrm{ml} / \mathrm{min} \text { per } 1.73 \mathrm{~m}^{2}\right)\end{array}$ & $1.03(0.99-1.06)$ & 0.134 & $1.04(1.00-1.08)$ & 0.037 & $1.03(0.99-1.07)$ & 0.144 \\
\hline Crescents $\geq 50 \%(v s<50 \%)$ & $3.51(0.92-13.32)$ & 0.065 & & - & $3.27(0.76-14.01)$ & 0.111 \\
\hline $\begin{array}{l}\text { Endocapillary hypercellularity } \\
\text { (E1 vs E0) }\end{array}$ & - & & $8.92(1.56-51.16)$ & 0.014 & $8.91(1.47-53.88)$ & 0.017 \\
\hline $\begin{array}{l}\text { Tubular atrophy/interstitial } \\
\text { fibrosis (T1/2 vs T0) }\end{array}$ & - & & $10.66(1.70-66.73)$ & 0.011 & $8.74(1.40-54.38)$ & 0.020 \\
\hline
\end{tabular}

Model 1: adjusted for proteinuria, hypertension, estimated glomerular filtration rate, and crescents.

Model 2: adjusted for proteinuria, hypertension, estimated glomerular filtration rate, endocapillary hypercellularity, and tubular atrophy/ interstitial fibrosis.

Model 3: adjusted for proteinuria, hypertension, estimated glomerular filtration rate, crescents, endocapillary hypercellularity, and tubular atrophy/interstitial fibrosis.

outcome after adjustment of proteinuria, hypertension, and estimated glomerular filtration rate (hazrad ratio $=3.51 ; 95 \%$ confidence interval $=0.92-$ 13.32; $P=0.065)$. Crescents were replaced by $E$ and $\mathrm{T}$ lesions in model 2, where hazard ratios for E1 and T1/T2 were 8.92 (95\% confidence interval $=1.56-$ 51.16; $P=0.014)$, and $10.66 \quad(95 \%$ confidence interval $=1.70-66.73 ; P=0.011$ ), respectively. When crescents, E, and T together were entered in model 3, E1 (hazard ratio $=8.91 ; 95 \%$ confidence interval = 1.47-53.88; $P=0.017$ ) and T1/T2 (hazard ratio $=8.74$; $95 \%$ confidence interval $=1.40-54.38 ; \quad P=0.020$ ) were independently associated with reaching the primary end point, while crescents were not.

\section{Discussion}

Henoch-Schönlein purpura nephritis and IgA nephropathy are currently considered to be different clinical presentations of the same disease. ${ }^{4-6}$ Similar to IgA nephropathy, increased levels of galactosedeficient IgA1 (Gd-IgA1) are detected in patients with Henoch-Schönlein purpura complicated by nephritis, and Gd-IgA1 is now believed to have a pivotal role in the pathogenesis of both IgA nephropathy and Henoch-Schönlein purpura nephritis. ${ }^{15,25}$ In addition, IgA-containing complexes have been found in the glomeruli of IgA nephropathy and Henoch-Schönlein purpura nephritis patients. Furthermore, we previously showed that HenochSchönlein purpura nephritis and IgA nephropathy had comparable prognoses when matched at presentation using propensity score analysis. ${ }^{6}$ Despite such similarities, classification of these two clinical entities has been based on different methods: the International Study of Kidney Disease in Children classification for Henoch-Schönlein purpura nephritis and either the Haas classification ${ }^{13}$ or the
Oxford classification for IgA nephropathy. In fact, the International Study of Kidney Disease in Children classification is based primarily on the presence of crescents, with much less emphasis on other pathologic features associated with adverse outcomes. In this regard, the need for a new classification system has been raised. ${ }^{9,15,26}$ Considering that Henoch-Schönlein purpura nephritis and IgA nephropathy have common features of pathogenesis and histopathologic findings, we hypothesized that the Oxford classification could also help predict long-term outcomes in HenochSchönlein purpura nephritis. Therefore, in this retrospective study, we evaluated for the first time the usefulness of the Oxford classification for patients with Henoch-Schönlein purpura nephritis.

In the present study, we found endocapillary hypercellularity and tubular atrophy and interstitial fibrosis to have an unfavorable influence on renal survival. The significance of their prognostic value persisted even after adjustment of well-known predictors such as baseline proteinuria and estimated glomerular filtration rate. It is of no surprise that tubulointerstitial lesions are strongly associated with adverse renal outcomes, as they are regarded as 'point of no return'. In general, many kidney diseases with high degrees of tubular atrophy/interstitial fibrosis on initial biopsy exert faster decline in kidney function over time. Of note, in patients with IgA nephropathy, T lesions of the Oxford classification system have been consistently been associated with poor renal outcomes in many validation studies of this classification. ${ }^{19-22}$ Furthermore, chronic lesions such as tubulointerstitial fibrosis have been identified as portending a poor prognosis in patients with Henoch-Schönlein purpura nephritis. ${ }^{10,11,26}$ In light of these data, the extent of tubulointerstitial lesions as a pathologic criterion should be included in future classification systems. 
We have also shown that endocapillary hypercellularity is associated with adverse renal outcomes. In addition, patients with endocapillary hypercellularity had greater baseline proteinuria than those without this lesion. Glomerular endothelial cells constitute part of the filtration barrier, and proteinuria occurs when the integrity of this framework is compromised. However, although endocapillary proliferation is considered to be a marker of acute glomerular inflammation, its prognostic value varies with the type of glomerulonephritis. ${ }^{26}$ In fact, endocapillary proliferation in lupus nephritis informs physicians that the patient may need urgent intensive immunosuppression because prognosis is poor if left untreated. ${ }^{27,28}$ In contrast, endocapillary proliferation in post-streptococcal glomerulonephritis generally does not portend a poor outcome because the kidney function of patients with post-streptococcal glomerulonephritis eventually recovers with little impairment. ${ }^{29}$ There has been very little research on endocapillary proliferation in Henoch-Schönlein purpura nephritis. Consistent with our findings, Pillebout et $a l^{10}$ showed that endocapillary proliferative lesions were significantly associated with an increased risk of severe renal failure by univariate analysis. An analogy can be made using relevant data from IgA nephropathy, but its significance has not yet been determined. A recent study showed that IgA nephropathy with diffuse endocapillary proliferation correlated with a better prognosis in patients with normal renal function, but it was a relative poor risk factor for end-stage renal disease in patients with abnormal renal function. ${ }^{30}$ Therefore, further studies are required to elucidate the prognostic value of endocapillary proliferation in Henoch-Schönlein purpura nephritis.

As rapidly progressive IgA nephropathy, which is characterized by abundant crescents, is rare, the presence of crescents was not selected for the Oxford classification. ${ }^{16,17}$ Crescents are much more often observed in Henoch-Schönlein purpura nephritis than in IgA nephropathy, and their number has been related to the severity of clinical signs and the prognosis of Henoch-Schönlein purpura nephritis in previous studies. ${ }^{5,7,11,26}$ In our study, crescents in $\geq 50 \%$ of glomeruli were more commonly observed in progressors and were significantly associated with reaching the primary end point in an unadjusted model (data not shown), whereas the significance was attenuated in the multivariable model. This finding supports a rationale for the limited utility of crescentic lesioins as a sole prognisticator. It is possible that a substantial proportion of crescents may eventually result in glomerular scarring, which is associated with decline in kidney function. However, there is an emerging concern that crescents, which are presumably transient, do not represent irreversible damage in patients with Henoch-Schönlein purpura nephritis, and thus may not be predictive of long- term outcome..$^{9,15}$ In fact, crescents, particularly when they are predominantly cellular, can resolve without progression to permanent scar tissue. ${ }^{15,31}$ In this regard, the predictive value of crescents in Henoch-Schönlein purpura nephritis does not appear to be potent. Of note, correlation between endocapillary proliferation and crescent formation has been suggested in Henoch-Schönlein purpura nephritis and IgA nephropathy, ${ }^{5,26,30}$ suggesting that these two lesions occur during the active inflammation stage. Whether such acute inflammatory lesions may have long-term impact remains to be further elucidated.

In our study, baseline proteinuria, which is classically known as a predictor of poor prognosis for many kidney diseases, was not a significant risk factor for progression to kidney failure. This finding is in contrast to the results from several previous studies showing that a large amount of proteinuria predicts adverse outcomes. ${ }^{10,11,32}$ Interestingly, Coppo et $a l^{9}$ suggested that proteinuria at the onset of Henoch-Schönlein purpura nephritis reflects acute inflammation and potentially reversible damage, thus decreasing its predictive value on long-term renal survival. This hypothesis could explain the lack of association between baseline proteinuria and renal outcome in our study. In fact, we found that proteinuria decreased in the study subjects from $1.69 \pm 2.27$ to $0.54 \pm 0.92 \mathrm{~g} /$ day during follow-up (data not shown), further supporting that proteinuria at the onset of Henoch-Schönlein purpura nephritis may not be a reliable predictor of progression. In addition, this study did not show whether renin-angiotensin system blockers or immunosuppressive drugs improved outcomes (data not shown). This can be attributed to the lack of established therapeutic guidelines and the retrospective nature of our study. In general, these drugs are prescribed if patients have substantial proteinuria and/or show progressive decline in kidney function. Thus, it is possible that, in our study, progressors received more renin-angiotensin system blockers or immunosuppressive drugs than nonprogressors. Indeed, there were more patients with nephrotic-range proteinuria $>3.0 \mathrm{~g} /$ day and those with crescents $\geq 50 \%$ in progressors. Nevertheless, although corticosteroids and cytotoxic drugs have been shown to be effective in active renal as well as extrarenal disease in Henoch-Schönlein purpura nephritis, the role of immunosuppressive drugs in long-term renal prognosis is not yet clearly defined. ${ }^{15}$

Our study has several other limitations. First, there are obvious differences in the histopathologic features between Henoch-Schönlein purpura nephritis and IgA nephropathy, although the two entities exhibit similar clinical and immunological features. Histologically, necrotizing glomerular lesions, diffuse endocapillary proliferation, and crescents with glomerular-fibrin deposits are more typical of Henoch-Schönlein purpura nephritis. ${ }^{5}$ Therefore, 
the application of the Oxford classification to Henoch-Schönlein purpura nephritis in clinical practice should be performed with caution and after appropriate modification. Second, our median follow-up duration was 49.3 months, which is relatively shorter than other studies. ${ }^{9-11}$ Given that Henoch-Schönlein purpura nephritis displays diverse patterns of progression to chronic kidney disease (scar from acute limited episodes or a slowly progressive active process as in IgA nephropathy), a longer period of observation is required to validate our findings. Finally, the observational nature of this study is a critical limitation. Some baseline covariates, including treatment and unmeasured characteristics, might not be distributed equally, and this could have led to biased results. In addition, our small sample size may limit generalizability into the whole population.

In summary, in our application of the Oxford classification to patients with Henoch-Schönlein purpura nephritis, we found endocapillary hypercellularity and tubular atrophy/interstitial fibrosis to be independent prognostic factors. This suggests that the Oxford classification can be used to predict adverse outcomes in Henoch-Schönlein purpura nephritis. The significance of crescentic lesions remains to be further explored, as we found its prognostic value to be attenuated in multivariate analysis. Our novel findings may provide a better histopathological implication of Henoch-Schönlein purpura nephritis with regards to estimating prognosis and may also emphasize the importance of establishing a new comprehensive histologic classification that can predict long-term outcomes. A larger multicenter study with a longer follow-up period is warranted to further clarify the predictive value of the pathologic criteria of Oxford classification in Henoch-Schönlein purpura nephritis.

\section{Acknowledgments}

This study was supported by a faculty research grant of Yonsei University College of Medicine for 2012 (6-2012-0033); and by a grant of the Korea Healthcare Technology R\&D Project, Ministry of Health and Welfare, Republic of Korea (HI10C2020).

\section{Disclosure/conflict of interest}

The authors declare no conflict of interest.

\section{References}

1 D'Amico G. Natural history of idiopathic IgA nephropathy and factors predictive of disease outcome. Semin Nephrol 2004;24:179-196.

2 Boyd JK, Cheung CK, Molyneux K, et al. An update on the pathogenesis and treatment of IgA nephropathy. Kidney Int 2012;81:833-843.
3 Evans DJ, Williams DG, Peters DK, et al. Glomerular deposition of properdin in Henoch-Schonlein syndrome and idiopathic focal nephritis. Br Med J 1973;3:326-328.

4 Waldo FB. Is Henoch-Schonlein purpura the systemic form of IgA nephropathy? Am J Kidney Dis 1988;12: 373-377.

5 Davin JC, Ten Berge IJ, Weening JJ. What is the difference between IgA nephropathy and HenochSchonlein purpura nephritis? Kidney Int 2001;59: 823-834.

6 Oh HJ, Ahn SV, Yoo DE, et al. Clinical outcomes, when matched at presentation, do not vary between adultonset Henoch-Schonlein purpura nephritis and IgA nephropathy. Kidney Int 2012;82:1304-1312.

7 Goldstein AR, White RH, Akuse R, et al. Long-term follow-up of childhood Henoch-Schonlein nephritis. Lancet 1992;339:280-282.

8 Niaudet P, Habib R. Schonlein-Henoch purpura nephritis: prognostic factors and therapy. Ann Med Interne (Paris) 1994;145:577-580.

9 Coppo R, Andrulli S, Amore A, et al. Predictors of outcome in Henoch-Schonlein nephritis in children and adults. Am J Kidney Dis 2006;47:993-1003.

10 Pillebout E, Thervet E, Hill G, et al. Henoch-Schonlein Purpura in adults: outcome and prognostic factors. J Am Soc Nephrol 2002;13:1271-1278.

11 Shrestha S, Sumingan N, Tan J, et al. Henoch Schonlein purpura with nephritis in adults: adverse prognostic indicators in a UK population. QJM 2006; 99:253-265

12 Ronkainen J, Nuutinen M, Koskimies O. The adult kidney 24 years after childhood Henoch-Schonlein purpura: a retrospective cohort study. Lancet 2002;360: 666-670.

13 Haas M. IgA nephropathy and Henoch-Schönlein purpura, In: Jennette JC, Olson JL, Schwartz MM, Silva FG(eds) Pathology of the Kidney, 6th edn. Lippincott Williams \& Wilkins: Phildelphia; 2007, pp 423-486.

14 Coppo R, Mazzucco G, Cagnoli L, et al. Long-term prognosis of Henoch-Schonlein nephritis in adults and children. Italian Group of Renal Immunopathology Collaborative Study on Henoch-Schonlein purpura. Nephrol Dial Transplant 1997;12:2277-2283.

15 Davin JC. Henoch-Schonlein purpura nephritis: pathophysiology, treatment, and future strategy. Clin J Am Soc Nephrol 2011;6:679-689.

16 Cattran DC, Coppo R, Cook HT, et al. The Oxford classification of IgA nephropathy: rationale, clinicopathological correlations, and classification. Kidney Int 2009;76:534-545.

17 Roberts IS, Cook HT, Troyanov S, et al. The Oxford classification of IgA nephropathy: pathology definitions, correlations, and reproducibility. Kidney Int 2009;76:546-556.

18 Coppo R, Troyanov S, Camilla R, et al. The Oxford IgA nephropathy clinicopathological classification is valid for children as well as adults. Kidney Int 2010;77: 921-927.

19 Alamartine E, Sauron C, Laurent B, et al. The use of the Oxford classification of IgA nephropathy to predict renal survival. Clin J Am Soc Nephrol 2011;6: 2384-2388.

20 Herzenberg AM, Fogo AB, Reich HN, et al. Validation of the Oxford classification of IgA nephropathy. Kidney Int 2011;80:310-317. 
21 Katafuchi R, Ninomiya T, Nagata M, et al. Validation study of oxford classification of IgA nephropathy: the significance of extracapillary proliferation. Clin J Am Soc Nephrol 2011;6:2806-2813.

22 Shi SF, Wang SX, Jiang L, et al. Pathologic predictors of renal outcome and therapeutic efficacy in IgA nephropathy: validation of the oxford classification. Clin J Am Soc Nephrol 2011;6:2175-2184.

23 Fogo AB. Approach to renal biopsy. Am J Kidney Dis 2003;42:826-836.

24 Levey AS, Stevens LA, Schmid CH, et al. A new equation to estimate glomerular filtration rate. Ann Intern Med 2009;150:604-612.

25 Lau KK, Suzuki H, Novak J, et al. Pathogenesis of Henoch-Schonlein purpura nephritis. Pediatr Nephrol 2010;25:19-26.

26 Szeto CC, Choi PC, To KF, et al. Grading of acute and chronic renal lesions in Henoch-Schonlein purpura. Mod Pathol 2001;14:635-640.
27 Markowitz GS, D'Agati VD. Classification of lupus nephritis. Curr Opin Nephrol Hypertens 2009;18:220-225.

28 Sprangers B, Monahan M, Appel GB. Diagnosis and treatment of lupus nephritis flares-an update. Nat Rev Nephrol 2012;8:709-717.

29 Eison TM, Ault BH, Jones DP, et al. Post-streptococcal acute glomerulonephritis in children: clinical features and pathogenesis. Pediatr Nephrol 2011;26:165-180.

30 Liu LJ, Li GT, Zhou Y, et al. Clinicopathologic features and outcomes in endocapillary proliferative IgA nephropathy. Nephron Clin Pract 2010;115:c161-c167.

31 Tipping PG, Kitching PR, Holdsworth SR. The formation of glomerular crescents, In: Neilson EG, Couser WG (eds) Immunologic Renal Diseases, 2nd edn. Lippingcott Williams \& Wilkins Publishers: Philadelphia, 2001.

32 Rauta V, Tornroth T, Gronhagen-Riska C. HenochSchoenlein nephritis in adults-clinical features and outcomes in Finnish patients. Clin Nephrol 2002;58:1-8. 